\title{
Analysis of Brain Functional MRI Time Series Based on Continuous Wavelet Transform and Stimulation-Response Coupling Distance
}

\author{
Laurent Thoraval ${ }^{1}$, Jean-Paul Armspach ${ }^{2}$, and Izzie Namer ${ }^{2}$ \\ 1 Laboratoire des Sciences de l'Image, de l'Informatique et de la Télédétection, \\ LSIIT/GRI - UPRES-A CNRS 7005, \\ ENSPS, Bd. Sébastien Brandt, F-67400 Illkirch, France \\ Laurent.Thoraval@ensps.u-strasbg.fr \\ http://lsiit.u-strasbg.fr \\ 2 Institut de Physique Biologique, \\ Faculté de Médecine - UPRES-A CNRS 7004, \\ 4, Rue Kirschleger, F-67085 Strasbourg Cedex, France \\ \{armspach, namer\}@ipb.u-strasbg.fr \\ http://ipb.u-strasbg.fr
}

\begin{abstract}
Analytical techniques applied to functional magnetic resonance imaging (fMRI) data require restrictive assumptions about the shape of the blood oxygenation level dependent (BOLD) time series observed at each voxel in response to a stimulation paradigm. In this paper, a radically different and fundamentally pattern recognition-oriented fMRI brain activation mapping approach is proposed. Neural activity is assessed at each voxel on a coupling distance principle between the deterministic alternation sequence of the stimulation paradigm and the sequence of BOLD response nonstationarities detected and characterized by a continuous wavelet transform (CWT). The voxel's stimulationresponse coupling distance is measured using an adapted version of the string edit distance algorithm. fMRI studies conducted on synthetic and real data demonstrated the superiority of the "coupling distance" brain activation mapping approach over against the Student's t-test.
\end{abstract}

\section{Introduction}

Through the BOLD effect that links neural activity to blood oxygenation levels in the vessels near active neurons, fMRI enables to image and study functional activity of the human brain. A typical fMRI experiment consists in alternating the acquisition of blocks of $N_{a}$ images $\left(N_{a} \sim 10\right)$ of the brain while the subject is in an active state, that is, performing a specific task, with the acquisition of blocks of $N_{c}$ images $\left(N_{c} \sim 10\right)$ while the subject is in a control state. This active-control or $\mathrm{ON}-\mathrm{OFF}$ cycle, when repeated $M$ times $(M \sim 10)$, constitutes the so-called stimulation paradigm.

Brain activation mapping consists in detecting regions of the brain activated by the stimulation paradigm. In standard practice, activation mapping is performed by means of the Student's t-test (TT), the cross-correlation (CC) or the 
statistical parametric mapping (SPM) approach. All three techniques are voxelwise: each BOLD response is analyzed independently. Neural activity is then declared at the voxel level if the corresponding BOLD response is determined by the stimulation paradigm somehow.

In essence, the TT measures a distance between two sample sets, one corresponding to the active fMRI samples, the other to the samples observed in the control state. The main drawback of the TT is that the time dimension of the BOLD fMRI signal is lost as the only information taken into account is the binary variable "stimulated" or "not stimulated". CC techniques are computationally efficient but rely on a priori knowledge about the reference waveform to be used. It can be either a boxcar function, a sine wave, or some mathematical model representative of the expected BOLD response [1]. The selected waveform can have a profound effect on the activation mapping results. Moreover, $\mathrm{CC}$ techniques consider the BOLD response as uniform across the brain. In the SPM approach 2], the BOLD response is fitted to a set of basis functions using a linear regression analysis. The basis functions are predefined according to the experimental protocol. They model either effects of interest that are sought or low frequency nuisance effects. A contrast map can then be obtained for any combination of the basis functions of interest with respect to the ones of no interest. SPM is a more poweful and versatile data processing approach than CC. But like CC, SPM requires morphological assumptions about the BOLD response which is unknown and may vary not only with the stimulation paradigm but also spatially within the brain.

In this paper, a new voxelwise brain activation mapping approach is developed that keeps the time dimension of the analysis while relaxing as much as possible traditional morphological assumptions about the ideal BOLD response expected at each voxel. Rather than considering the shape of the local BOLD response in its whole, the proposed approach focuses on its dynamics changes. In the presence of a regional neural activity, these are assumed to be time-locked to some extent onto the successive transitions of the stimulation paradigm.

\section{Methods}

A continuous wavelet transform (CWT) is first applied to each voxel's fMRI signal under test to detect and characterize dynamics changes of interest. The derived sequence of events, $R$, is then compared to the deterministic event sequence, $S$, that models the successive transitions of the stimulation paradigm. A "coupling distance", $\operatorname{dist}(R \rightarrow S)$, between the response $R$ and the stimulation $S$ is finally worked out using a dynamic event matching procedure.

\subsection{Detection / Characterization of fMRI Signal Changes by CWT}

Wavelet multiresolution analysis has been widely used in multiscale representation and analysis of signals and images [3]. The wavelet-based brain activation mapping approach presented here differs significantly from the pioneering work 
of Ruttimann et al. [4]. Rather than detecting neural activity based on a discrete wavelet transform of the average difference image from the input fMRI sequence as proposed by Ruttimann et al., neural activity is detected here based on the discrete space-time field of signal sharp variations underlying the raw 3-D fMRI sequence. The ability of the CWT to detect and characterize such nonstationarities in the time-scale domain is here of great interest. For any finite energy signal $f(t)$, the CWT is defined by $[3]$ :

$$
\left(W_{\psi} f\right)(a, b)=\frac{1}{\sqrt{a}} \int_{-\infty}^{+\infty} f(t) \psi^{*}\left(\frac{t-b}{a}\right) d t
$$

The parameters $a$ and $b$ denote the scale and the translation parameter respectively of the complex conjugate of the mother wavelet $\psi(t)$ where:

$$
\psi(t)= \begin{cases}C \cdot\left(1+\cos 2 \pi f_{0} t\right) \cdot e^{2 i \pi k f_{0} t} & \text { for }|t| \leq \frac{1}{2 f_{0}} \\ 0 & \text { elsewhere }\end{cases}
$$

$\mathrm{k}$ is set to 2 to satisfy with the required admissibility conditions while $f_{0}$ denotes the normalized frequency $\left(0<f_{0}<1 / 2\right)$ and $C$ a normalizing constant. $\psi(t)$ is chosen complex valued to benefit from the phase information of the fMRI signal CWT. Indeed, it has been demonstrated in [3] that symmetry singularities of a signal $f(t)$ are associated with particular values of the phase of its CWT. Specifically, a positive inflexion point at time $t_{0}\left(f^{\prime \prime}\left(t_{0}^{-}\right)>0, f^{\prime \prime}\left(t_{0}\right)=\right.$ $\left.0, f^{\prime \prime}\left(t_{0}^{+}\right)<0, f^{\prime}\left(t_{0}\right)>0\right)$ is associated at high resolution (i.e., small values of the scale parameter " $a$ ") with phase value $+\pi / 2$. Similarly, a negative inflexion point at time $t_{0}\left(f^{\prime \prime}\left(t_{0}^{-}\right)<0, f^{\prime \prime}\left(t_{0}\right)=0, f^{\prime \prime}\left(t_{0}^{+}\right)>0, f^{\prime}\left(t_{0}\right)<0\right)$ corresponds at high resolution to phase value $-\pi / 2$. Then, assuming that the fMRI dynamics changes of interest exhibit a local positive or negative inflection point, detecting the occurrence times of these changes can be performed at a sufficient high scale of resolution by detecting the $\pm \pi / 2$ crossings of the phase of the fMRI signal CWT. Moreover, while moving towards finest scales of analysis, $\pm \pi / 2$ crossing points are increasing while describing curves known as fingerprints in pattern recognition. The length of a fingerprint, expressed in terms of the number of scales crossed from the finest scale of detection, is characteristic of the importance of the fMRI dynamics change associated with. Finally, as stated in [3], $\pm \pi / 2$ crossing points correspond to maxima of the square modulus of the fMRI signal CWT.

As an example, the phase of the CWTs of an activated and a non-activated fMRI signal are plotted in figure 1. The horizontal time axis is indexed by the scan number in the fMRI sequence while the scale index $i$ is reported on the vertical axis of figures $1 k-\mathrm{d}$. The frequency $f_{0}=0.04$ and the scale parameters are $a_{i}=f_{0} /\left(f_{0}-|i-1| . \Delta\right)$, with $\Delta=0.002,1 \leq|i| \leq 15$. Color jumps from white to black correspond to $+\pi / 2$ crossings for $i \leq-1$ and $-\pi / 2$ crossings for $i \geq 1$ so that the color jumps at the virtual scale $i=0$ correspond to the occurrence times of the detected dynamics changes of interest.

In practice, a feature vector $\mathbf{v}=\left(v_{1}, \cdots, v_{K}\right)$ is derived for each dynamics change detection point. Two features are retained, namely, $v_{1}$, the length of the 


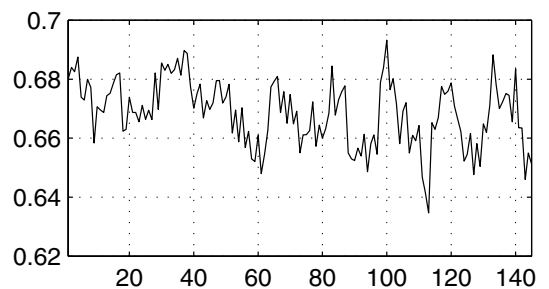

a) Activated fMRI signal

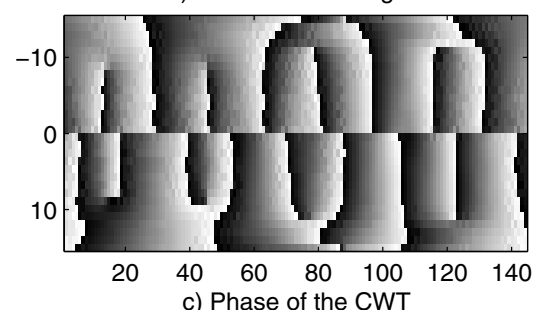

c) Phase of the CWT
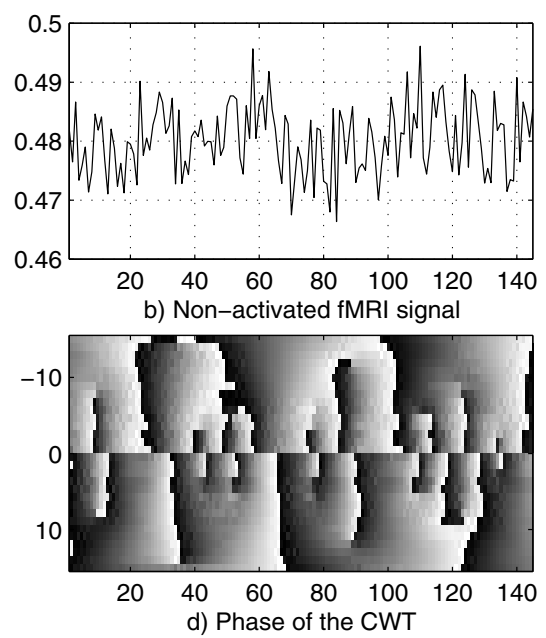

Fig. 1. Phase information of real fMRI data CWT.

corresponding fingerprint, starting at scale $i= \pm 1$ and corresponding to the integer number of associated levels of decomposition, and $v_{2}$, the sum of the square modulus along the fingerprint. Then, the event $r=(t, p, \mathbf{v})$ is formed by appending $\mathbf{v}$ to the pair $(t, p)$ of integers where $t$ and $p$ denote the occurrence time and the "polarity" of the change, respectively. That is, $p$ takes the value \pm 1 according to the $\pm \pi / 2$ value of the phase crossing at $t$. Finally, an ordered event sequence $R=r_{1}, r_{2}, \cdots, r_{I}$ of length $|R|=I$ is produced for every fMRI signal.

\subsection{Modeling of the Stimulation Paradigm Dynamics}

Dynamics modeling of a two states-based stimulation paradigm is straightforward. Every transition is modeled by an event $s=\left(t^{\prime}, p^{\prime}\right)$ where $t^{\prime}$ and $p^{\prime}$ represent the occurrence time and the "polarity" of the transition in the stimulation paradigm, respectively. As $p, p^{\prime}$ takes the arbitrary +1 or -1 value according to the OFF-ON or ON-OFF nature of the transition, respectively. Thus, the stimulation paradigm dynamics is modeled by the deterministic event sequence $S=s_{1}, s_{2}, \cdots, s_{J}$ of fixed length $|S|=J$, where $s_{j}=\left(t_{j}^{\prime}, p_{j}^{\prime}\right)$ models the $j$-th transition of the paradigm. By contrast to the multiple response sequences, it can be noticed that the stimulation sequence $S$ is unique.

\subsection{Matching of Event Sequences}

The coupling distance between the stimulation sequence $S$ and a response sequence $R$ has been developed based on the string edit distance used in text processing [56]. First, "matching" operations are introduced in place of the wellknown "edit" operations. A matching operation $m$ is a pair $(x \rightarrow y) \neq(\emptyset \rightarrow \emptyset)$ 
where $x(y)$ denotes either an event $r_{i}$ of $R$ (an event $s_{j}$ of $S$ ) or the NULL event $\emptyset$, in place of the NULL string. Three matching operations can then be defined, namely, the insertion (ins), the deletion (del) and the valid matching $(v m)$ which take the general forms $\left(\emptyset \rightarrow s_{j}\right),\left(r_{i} \rightarrow \emptyset\right),\left(r_{i} \rightarrow s_{j}\right)$, respectively.

As in the string edit distance, a specific cost $c(m)$ is assigned to each matching operation $m \in\{i n s, d e l, v m\}$. An insertion can be considered as a misdetection of a fMRI dynamics change of interest in response to the paradigm transition $s_{j}$. Similarly, a deletion can be viewed as a false alarm. Defining the cost of a valid matching operation is less straightforward for many reasons. First, matching a fMRI dynamics change with a paradigm transition of opposite polarity is clearly unnatural and must be unvalidated as such. Second, one will assign a cost all the more low as the dynamics change to be matched is important in terms of feature magnitudes. Third, an activation delay $d$ at a particular voxel has to be modeled. Based on these remarks, the costs of an insertion, a deletion and a valid matching operation are respectively defined by $c($ ins $)=w_{m d}, c($ del $)=w_{f a}$, and $c(v m \mid d)=$ $\alpha_{0} \cdot c_{0}\left(d, t_{i}, t_{j}^{\prime}\right)+\sum_{k=1}^{K} \alpha_{k} \cdot c_{k}\left(v_{k}\right)$ where the misdetection weight $w_{m d}$ and the false alarm weight $w_{f a}$ are preset constants. $\left\{\alpha_{k}\right\}$ are weighting coefficients verifying $\sum_{k=0}^{K} \alpha_{k}=1$ while $\left\{c_{k}().\right\}$ are positive cost functions selected ad hoc and upper bounded $\left(\sup \left(c_{k}().\right)=w_{\max }, \forall k\right)$. For any valid matching $v m, w_{\max }$ must verify $w_{\max } \leq w_{m d}+w_{f a}$. Indeed, depending on the feature magnitudes, if $w_{\max }>$ $w_{m d}+w_{f a}$, it could be never useful to match two events, one can always delete and insert an event, instead. The cost functions used in our approach are simple and are plotted in figure 2. $W$ models the detected change occurrence time
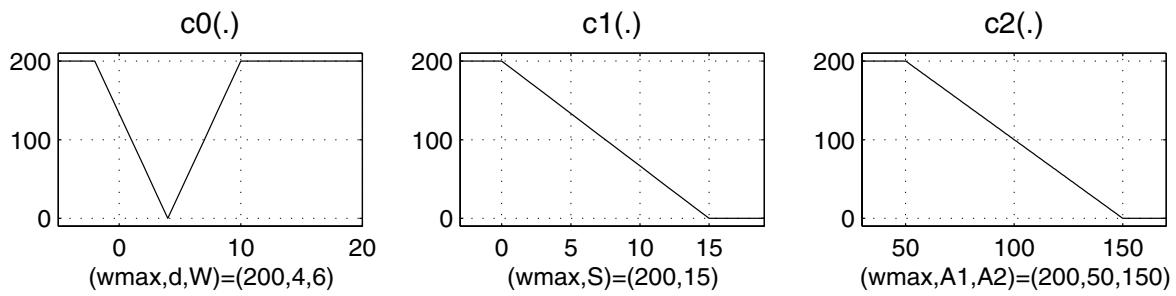

Fig. 2. Cost functions used for a valid event matching.

jitter allowed around the given delay $d . S$ denotes the highest scale index of the CWT. $A 1$ and $A 2$ are real parameters arbritarily set to the $\mu-\sigma$ and $\mu+\sigma$ values respectively where $\mu$ and $\sigma$ represent the mean and the standard deviation of the feature $v_{2}$ estimated from the input voxel's sequence $R$.

Now, to measure the coupling distance between the stimulation sequence $S$ and a response sequence $R$, one will typically need to successively apply different event matching operations. Let $M=m_{1}, \cdots, m_{l}, \cdots, m_{L}$ be a matching sequence of length $L$ where $m_{l} \in\{i n s, d e l, v m\}$. Then, the cost of $M, c(M)$, is defined as the sum of the costs of the elementary matching operations $m_{l}$ making up the sequence, that is, $c(M)=\sum_{l=1}^{L} c\left(m_{l}\right)$. Given $R$ and $S$, there may be more than one 
matching sequence $M$ that couples $R$ to $S$. If $M_{R \rightarrow S}$ denotes the set of all such sequences, then the coupling distance $\operatorname{dist}(R \rightarrow S)$ is given by the matching sequence with the minimum cost, that is, $\operatorname{dist}(R \rightarrow S)=\min \left[c(M) \mid M \in M_{R \rightarrow S}\right]$. In practice, $\operatorname{dist}(R \rightarrow S)$ is computed based on dynamic programming [5]. Given the activation delay $d \in D=\left[d_{\text {min }}, d_{\text {max }}\right]$, let $\delta(i, j, d)$ denote the partial minimum cost needed to match the first $i$ events of $R$ with the first $j$ events of $S . \delta(i, j, d)$ is computed as follows:

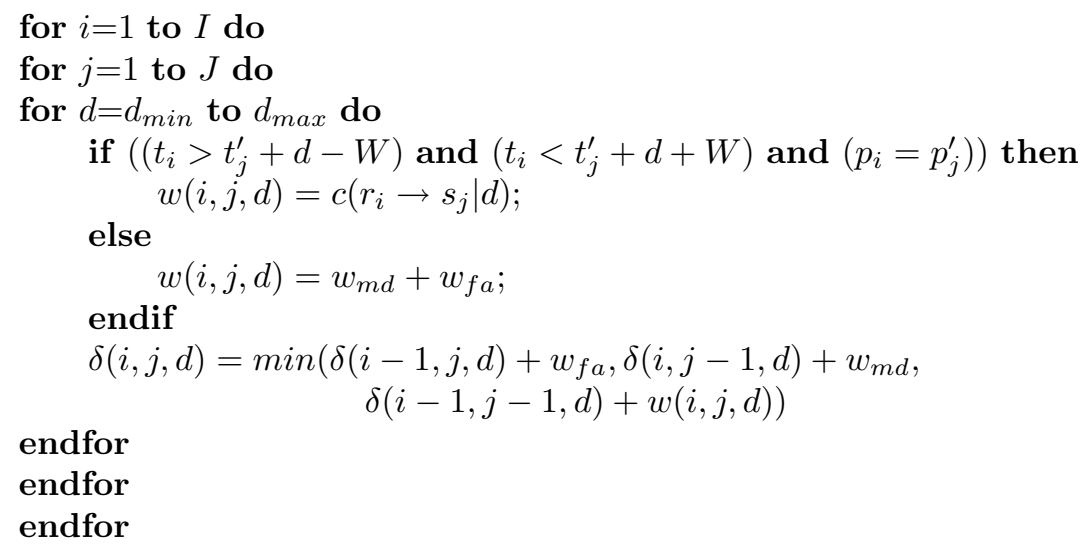

The initialisations are $\left.i) \delta(0,0, d)=0, \forall d, i i) \delta(i, 0, d)=i . w_{f a}, \forall i, i i i\right) \delta(0, j, d)=$ $j . w_{m d}, \forall j$. Then, the coupling distance $\operatorname{dist}(R \rightarrow S)$ is obtained by $\operatorname{dist}(R \rightarrow$ $S)=\min _{d \in D} \delta(I, J, d)$, where $I=|R|$ and $J=|S|$ as stated previously.

\section{Results and Discussion}

A synthetic fMRI data set was first generated. To simulate distinct activated BOLD responses, a square wave, a sinus wave and a periodic Poisson signal in additive white gaussian noise were used. The period of each waveform was set to $T=16\left(N_{a}=N_{c}=M=8\right)$ so that $|S|=J=16$ given a 145 fMRI samples time-series. The fluctuation of the synthetic BOLD response with respect to the constant baseline of magnitude 0.5 was set to $\pm 10 \%$. The noise variance was set so that the $\mathrm{SNR}=20 \mathrm{~dB}$ in the overall experiment. To simulate activation delay, the phase had a spatial random fluctuation uniformly distributed over $T / 3$, along with a constant phase shift for any given activated voxel's timeseries. The lag parameter of the Poisson waveform was arbitrarily set to $\lambda=5$. The parameters used for the CWT were those mentionned in section 2.1. As a preliminary evaluation, the parameters of the coupling distance algorithm were set to $\left(w_{m d}, w_{f a}, w_{\max }\right)=(100,100,200)$ and $\left(W, d_{\min }, d_{\max }\right)=(4,-2,13)$ in accordance with the period $T$. Synthetic neural activity detection results obtained by the proposed method have been compared to those obtained by the Student's t-test. The corresponding ROC curves are plotted in figure 3 In all cases, the coupling distance method outperforms the t-test. However, the characterization 

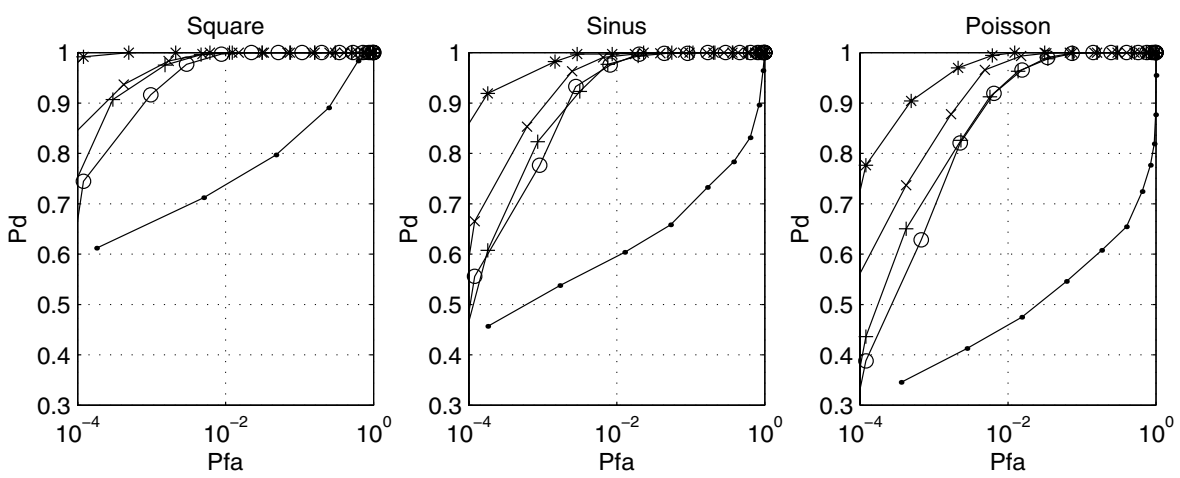

Fig. 3. Neural activity detection ROC curves obtained from synthetic fMRI data by the t-test (.) and the coupling distance method with $\left(\alpha_{0}, \alpha_{1}, \alpha_{2}\right)=(1,0,0)(*)$, $(.5, .5,0)(+),(.5,0, .5)(\mathrm{x}),(.34, .33, .33)(\mathrm{o})$.

of the detected dynamics changes appears inadequate since the ROC curves for $\left(\alpha_{0}, \alpha_{1}, \alpha_{2}\right)=(1,0,0)$ remain all maximum compared with other triplets of values.

Language fMRI studies have also been conducted. Images were acquired with a $2 \mathrm{~T}$ whole body S200 Bruker MRI system with a head volume coil. fMRI images were obtained with echo-planar imaging (EPI) using an axial slice orientation (32 slices, $64 \times 64$ pixels, voxel size $=4 \times 4 \times 4 m m, T E=10 \mathrm{~ms}, \mathrm{TR}=5 \mathrm{~s}$ ). All fMRI images were registered to the first image in the series. Three testing procedures were designed to determine the cortical areas involved in word finding, auditory and visual lexical processing. Activation maps obtained for the verb generation task are compared in figure 4. Coupling distance activation maps demonstrated all together the ability of the method to detect additional activated regions with respect to the standard t-test. From a signal processing point of view, trueactivated fMRI signals declared non-activated by the t-test where essentially corrupted by impulsive noise or baseline drift, or exhibited activation delays along with low SNRs.

\section{Conclusion and Future Work}

A new method for detecting activated voxels in fMRI brain activation studies has been presented. Unlike other methods, no a priori knowledge is required about the shape of the BOLD response expected at each voxel. In the presence of neural activity, BOLD response dynamics changes are assumed to be time-locked to some extent onto the active-control transitions of the stimulation paradigm. Time-locking is expressed in terms of a coupling distance between the sequence of dynamics changes of interest detected and characterized by a CWT and the deterministic transition sequence of the stimulation paradigm. The coupling distance is computed based on a modified version of the string edit 

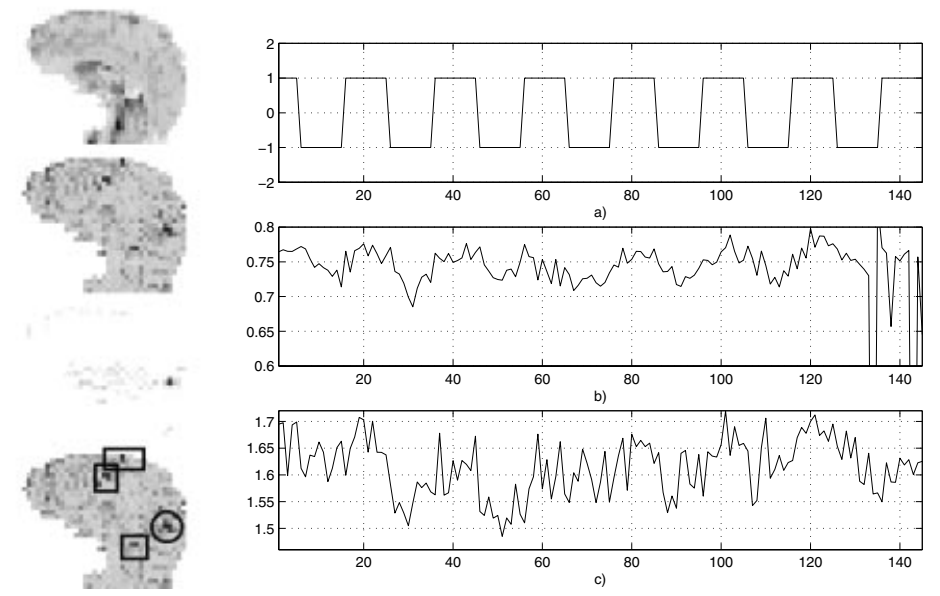

Fig. 4. Results on real fMRI data. Left side, from top to bottom: fMRI slice, coupling distance activation map, t-test activation map, copy of the coupling distance activation map with one common (circle) and three additional (rectangles) activated regions detected. Right side: a) paradigm transitions, b-c) two activated fMRI signals picked up from the rectangles of the coupling distance activation map.

distance algorithm used in text processing. The method has been compared to the standard Student's t-test on synthetic and real fMRI data. Results obtained in both cases demonstrated the superiority of the "coupling distance" approach.

The proposed method has now to be compared further. In addition, we plan to introduce spatial information in the measure of the coupling distance by reformulating the approach in terms of multiple dynamics change sequence alignment.

\section{References}

1. Bandettini, P.A., Jesmanowicz, A., Wong, E.C., Hyde, J.S.: Processing strategies for time-course data sets in functional MRI of human brain. Magn. Reson. Med., 30 (1993) 161-173

2. Friston, K.J., Jezzard, P., Turner, R.: Analysis of functional MRI time-series. Human Brain Mapping, 1 (1994) 153-171

3. Aldroubi, A., Unser M.: Wavelets in Medicine and Biology. CRC Press (1996)

4. Ruttimann, U.E., Unser, M., Rawlings, R.R., Rio, D., Ramsey, N.F., Mattay, V.S., Hommer, D.W., Frank, J.A., Weinberger, D.R.: Statistical Analysis of Functional MRI Data in the Wavelet Domain. IEEE Trans. Med. Imaging, 17 (1998) 142-154

5. Wagner, R.A., Fischer, M.J.: The string-to-string correction problem. J. Assoc. Comput. Machinery, 21 (1974) 168-173

6. Marzal, A., Vidal, E.: Computation of normalized edit distances and applications. IEEE Trans. Pat. Ana. Mach. Int., 15 (1993) 926-932 